\title{
EFECTOS DEL PASTOREO EN RIQUEZA FLORÍSTICA, BIOMASA Y COBERTURA DE UN PASTIZAL DE ALBARDÓN, ARGENTINA
}

\author{
Ingrid Irene Kaufmann', Susana Raquel Feldman²; ${ }^{2}$ Mónica B. Sacido²
}

${ }^{1}$ Investigadora en EEA Alto Valle, Instituto Nacional de Tecnología Agropecuaria, Ruta 22 Km 1190. C.P.: 83332, Allen, Río Negro, Argentina.

${ }^{2}$ Facultad Cs Agrarias, Universidad Nacional de Rosario.

${ }^{3}$ CIUNR \& IICAR (Instituto de Investigaciones en Ciencias Agrarias de Rosario)

Email: kaufmann.ingrid@inta.gob.ar

\begin{abstract}
RESUMEN
Se analizó el efecto del pastoreo y descansos sobre la riqueza florística, la producción de materia seca y la cobertura de un pastizal degradado en el Delta del Paraná. El estudio abarcó 13 meses, divido en un primer sub-período con tres tratamientos: pastoreo continuo con alta carga (1 equivalente vaca/ha), clausura con cortes bimestrales de la vegetación y clausura sin cortes, y segundo sub-período con: anegamiento y sin clausuras. Los datos de vegetación se analizaron mediantes métodos multivariados. Según los resultados, en contraposición al pastoreo continuo, con descansos de dos meses se cubrió totalmente el suelo, hubo un incremento en la riqueza florística nativa, aparecieron especies forrajeras de crecimiento primavero- estivootoñal y en dicha estación de crecimiento mejoró la oferta de materia seca un $22,5 \%$. Es posible favorecer valores de las variables estudiadas del pastizal y mejorar la oferta forrajera con un manejo adecuado.

Palabras clave: cobertura del suelo, descansos, oferta forrajera, pastoreo, riqueza florística.
\end{abstract}

Recibido: 10 de Octubre de 2018. Aceptado: 08 de Enero de 2019

Received: October 10, 2018. Accepted: January 08, 2019

\section{EFFECTS OF GRAZING IN THE FLORISTIC WEALTH, BIOMASS AND COVERAGE OF AN ALBARDON PASTURELAND, ARGENTINA}

\begin{abstract}
The effects of grazing and breaks resting were analyzed regarding the floristic richness, the production of the dry matter and forage coverage of a degraded pasture from the Parana River Delta. The study covered 13 months, divided into a first sub-period with three treatments: continuous grazing with high load (1 equivalent cow.ha-1), closure with bimonthly cuts of vegetation, and closure without cuts, and second sub-period with: waterlogging and without closures. Vegetation data was analyzed by multivariate methods. According to the results, in contrast to continuous grazing, two-month rests covered completely the soil, increased native species richness, forage species of spring-summer-autumnal growth appeared and improved the offering of dry-matter in this growing season by $22,5 \%$. It is possible to favor values of the studied variables of the pasture and to improve the forage offer with proper management.

Keywords: soil cover, breaks, forage offer, grazing, floristic richness.
\end{abstract}

Cómo citar este artículo: I. Kaufmann, S. Feldman, M. Sacido. "Efectos del pastoreo en riqueza florística, biomasa y cobertura de un pastizal de Albardón, Argentina”, Revista Politécnica, vol. 15, no.29 pp.95-107, 2019. DOI: 10.33571/rpolitec.v15n29a8 


\section{INTRODUCCIÓN}

El Delta del Río Paraná forma parte del Sistema de Humedales Paraguay-Paraná, el corredor de humedales más importante del mundo, que se extiende desde el Pantanal de Mato Grosso (Brasil) hasta el Río de la Plata (Argentina y Uruguay) [1]. El Delta propiamente dicho está ubicado en los últimos $320 \mathrm{~km}$ del río, entre las latitudes $32^{\circ} 5^{\prime}$ y $34^{\circ} 29^{\prime} \mathrm{S}$, con una superficie de $17.500 \mathrm{~km}^{2}$ [2]. El área posee un perfil diferencial de riqueza de hábitats [3], que se sostiene por la complejidad espacial y heterogeneidad ambiental, proporcionada por sus características de formación, ubicación y moldeada por la peculiar hidrología del territorio [1]. Las islas se caracterizan por tener la forma semejante a una palangana irregular, con el margen alto, llamado albardón y el interior bajo, zona de bañado pantanoso, con aguas estancadas y vegetación de pajonal [4].

Los bienes y servicios ecosistémicos que brinda el Delta del Paraná incluyen la provisión y filtrado de agua dulce, recarga de acuíferos, fertilidad de suelos, retención de nutrientes, regulación del clima, calidad del aire, control de plagas y enfermedades, disponibilidad de polinizadores, amortiguación de inundaciones, vegetación de uso medicinal, apícola, madera y frutas y secuestro de carbono en biomasa reduciendo el contenido de $\mathrm{CO}_{2}$ atmosférico [5].El origen de los suelos del Delta es aluvial y su material constitutivo y genético son los sedimentos de los ríos que componen la cuenca [6]. En general presentan una pobre estructuración y escaso desarrollo de perfiles [7]. Previo a la intensificación del uso de esta región, los herbívoros más destacados eran el ciervo de los pantanos (Blastocerus dichotomus) y el carpincho (Hydrochoerus hydrochaeris) [8].

Las características de este humedal, clima templado, agua apta para consumo animal y forraje con alta abundancia de especies nativas de excelente calidad [9] [10] han favorecido el desarrollo de la ganadería bovina, que hasta la fecha ha sido de tipo extensiva, con cargas fluctuantes pero generalmente demasiado altas, entre 0,5 y más de un equivalente vaca por ha $\left(E^{*} \mathrm{ha}^{-1}\right)$ [11]. Esto ha provocado cambios en la estructura de los pastizales y alteraciones en la dinámica poblacional de las especies más palatables [11] [12]. Según Quintana [5] los efectos en la estructura y composición del pastizal se asocian a diferencias en las cargas, registrando impactos negativos con altas cargas tanto en la biomasa aérea como subterránea, en la reproducción vegetal y en la riqueza específica entre otros factores, afectando el funcionamiento general del humedal respecto a situaciones con baja carga. En el caso de ambientes degradados, este autor recomienda la exclusión de la ganadería, para permitir que comience a restablecerse la integridad ecológica, y con ella la provisión de ciertos bienes y servicios ecosistémicos relevantes para la propia actividad ganadera, tales como ser el incremento de la cobertura y biomasa de especies de alta calidad forrajera. En estos pastizales los anegamientos periódicos son un disturbio intrínseco al ecosistema, que obliga a retirar al ganado produciendo un descanso obligado del pastoreo. En este sentido la actividad ganadera se realiza enmarcada en el contexto eco-hidrológico compatibilizando con la dinámica natural del Delta [13].

Por otro lado, los cambios posteriores a un disturbio podrían responder a la hipótesis de disturbio intermedio, la cual postula que la mayor diversidad se produce a intensidades intermedias de disturbio 0 en lapsos intermedios entre disturbios sucesivos [14] o la composición florística inicial, que sostiene que en una sucesión secundaria las etapas iniciales son las de la mayor diversidad, ya que los propágulos de las especies que se establecen en el transcurso de una sucesión secundaria, post-disturbio, estaban ya presente en la misma formando parte del banco de propágulos de la comunidad pre-disturbio [15].

Las especies vegetales presentes en un lugar difieren en sus respuestas a los cambios ambientales y a los disturbios, entre los cuales se pueden mencionar crecidas del río, sequías, heladas y pastoreo por parte de grandes herbívoros. Los efectos de los grupos funcionales repercuten directamente sobre las comunidades que se encuentran en el humedal, sobre las diferentes funciones de los ecosistemas y como consecuencia, sobre la provisión de bienes y servicios ecológicos aportados por éstos al desarrollo de las sociedades humanas [16] [17]. Estudios preliminares señalan que la ganadería afecta principalmente los sectores medios y altos del gradiente topográfico de las islas, modificando los pastizales por el consumo selectivo del pastoreo [18]. Altas cargas (1 equivalente vaca/ha), la selección, la falta de descansos, quemas reiteradas y el pisoteo del sustrato en condiciones de alta humedad conforman una dinámica de mal manejo del pastoreo que puede producir cambios negativos en la dinámica del agua y las sales del suelo [5]. Como consecuencia del sobrepastoreo, numerosos pastizales de albardón y caída de albardón, presentan baja riqueza florística, dominancia de la exótica naturalizada Cynodon dactylon [12], una disminución de la calidad del ambiente y, por ende, en la provisión de ciertos bienes y servicios ecosistémicos relevantes para la propia actividad ganadera [5]. 
En la zona del estudio se observaron especies graminiformes bajas (Bromus catharticus, Polypogon mospeliensis y Carex bonaerensis), además de no-graminiformes (Phyla canescens, Eryngiun echinatum y Polygonum punctatum).

El objetivo de este trabajo fue establecer los cambios en cobertura de suelo, disponibilidad de biomasa y riqueza florística de un pastizal de albardón con historia de sobrepastoreo, bajo dos regímenes de consumo (pastoreo continuo y cortes bimestrales que simulan un pastoreo intenso).

\section{MATERIALES Y METODOS}

El experimento se llevó a cabo en el pastizal de albardón del río Baradero, dentro de la planicie inundable del Delta del río Paraná, en el partido de Baradero, provincia de Buenos Aires, Argentina. Se presume que hace varias décadas hay presencia de ganado doméstico. Anteriormente lo habitaban herbívoros nativos, como el ciervo de los pantanos (Blastocerus dichotomus) y el carpincho (Hydrochoerus hydrochaeris) [9].

Los tratamientos se iniciaron el 10 de agosto 2012 y hasta el 13 de septiembre de 2013, en un pastizal con décadas de pastoreo intermitente con ganado bovino. El período de estudio fue dividido en dos etapas, la primera hasta junio de 2013 inclusive, y la segunda etapa abarcó julio, agosto y septiembre del mismo año. Los tratamientos fueron: C, clausura al pastoreo con cortes bimestrales de la vegetación a $7 \mathrm{~cm}(n=3 ; 4 \mathrm{~m} x$ $4 \mathrm{~m})$; S, clausura al pastoreo sin cortes $(n=3 ; 4 m x$ $4 \mathrm{~m}$ ); y $\mathrm{P}$, pastoreo continuo con altas cargas (1 equivalente vaca / ha). El tratamiento $P$ fue el pastizal por fuera de las clausuras, abarcando una superficie de $150 \times 50 \mathrm{~m}$, donde se tomaron muestras utilizando la misma metodología que en las clausuras. Los cortes del tratamiento $\mathrm{C}$ se hicieron bimestralmente entre agosto de 2012 y abril de 2013 y, en los casos que coincidían con tomas de muestras o censos de vegetación, con posterioridad a los mismos. Se decidió cortar/cosechar solo el material vegetal por arriba de los $7 \mathrm{~cm}$ de altura, siguiendo la recomendación general que sugiere Carámbula [19]. Como la temperatura es un factor muy influyente en la tasa de crecimiento del pastizal [20], la toma de muestras se distribuyó en estaciones agronómicas (fin invierno 2012, primavera 2012, verano 20122013, otoño 2013 y primavera 2013). Estas se establecieron de acuerdo a la evolución de las temperaturas medias diarias y a la concentración de heladas, considerando invierno los tres meses en los que se dio la mayor concentración de heladas, aproximadamente 20 días antes respecto a las estaciones calendarias. La segunda etapa comenzó el primero de julio de 2013, cuando la vegetación se expuso a un intenso pastoreo seguido por anaerobiosis, ya que ese invierno sobrevino una crecida de los ríos y anegamiento del pastizal -julio y agosto- que ameritó el desarme de las clausuras.

En cada estación agronómica se registraron y analizaron las siguientes variables:

Cobertura de suelo: Se realizó una estimación visual del porcentaje de suelo cubierto por vegetación, discriminando forrajeras, no forrajeras y suelo descubierto, dentro del aro de muestreo de $35,7 \mathrm{~cm}$ de diámetro $\left(0,10 \mathrm{~m}^{2}\right)$ en los tratamientos C y S. En el sector $P$ se muestreó al inicio y al final del experimento con el mismo método. Los datos se analizaron con Kruskal Wallis, utilizando Infostat [19], ya que esta prueba no paramétrica es equivalente a un análisis de varianza de una vía (ANOVA). Es válida de usar cuando no se cumplen determinados requisitos, como normalidad y homocedasticidad, prácticamente sin perder potencia en relación al análisis paramétrico (95\% del mismo) ${ }^{1}$.

Riqueza florística: Se censaron las especies presentes en todas las unidades experimentales $(n=3$ para $S$ y $C$ y en 2 transectas de 20 pasos en $8 \mathrm{~m}$ para el tratamiento $\mathrm{P}$ ), utilizando la escala de abundancia-cobertura [22], modificada según van der Maarel [23] y se analizaron mediante métodos multivariados, análisis de componentes principales (PCA por su denominación en inglés: principal component analysis), utilizando el programa informático PC-Ord [24]. La comunidad vegetal se caracterizó según criterios de origen (nativa o exótica) y de aptitud forrajera. La nomenclatura botánica es según Zuloaga et al. [25]. En el herbario del Instituto de Botánica Darwinion ${ }^{2}$ están coleccionados ejemplares de referencia de las especies registradas.

Altura promedio de la vegetación: Se promediaron las mediciones de la altura de la vegetación (en $\mathrm{cm}$ ) realizadas con regla milimetrada en 5 puntos tomados al azar dentro de cada unidad experimental (C, S y $\mathrm{P})$.

Materia seca disponible: Se determinó la biomasa presente por encima de los $7 \mathrm{~cm}$ de altura, en los 3 tratamientos y cada estación agronómica, en parcelas al azar, sin superponer. El material cosechado se separó en materia seca (MS) muerta y MS viva y ésta, en MS de especies forrajeras y de no forrajeras, se secó hasta peso constante en estufa a $65^{\circ} \mathrm{C}$, pesó en balanza de precisión y los datos se expresaron en $\mathrm{kg} \mathrm{MS} \mathrm{ha}^{-1}$. La tasa de crecimiento o de rebrote se calculó por diferencia de forraje disponible entre muestreos [26].

${ }^{1}$ https://rstudio-pubs-static.s3.amazonaws.com/219504 8f53aa50801b4b0a927e317844ecfec4.html

${ }^{2}$ http://www.darwin.edu.ar/Proyectos/FloraArgentina/Generos.a sp?Letra $=\mathrm{O}$ 


\section{RESULTADOS}

Los cortes, comparables a pastoreos cortos e intensos, no afectaron por igual a todas las especies presentes.

Cobertura de suelo: Al momento de colocar las clausuras (10 agosto 2012), la mitad del suelo estaba desnudo, casi un $30 \%$ cubierto con especies no forrajeras y solo el $21 \%$ con especies forrajeras. A los 20 días de haber clausurado se observó un incremento en la proporción de suelo cubierto con especies no forrajeras, mientras que el porcentaje de suelo desnudo (SD) bajó a $18 \%$, siendo las diferencias con el sector $\mathrm{P}$, sin clausurar y con pastoreo continuo, estadísticamente significativas (Kruskal Wallis, $\mathrm{p}=0.0357$ ), cuyos valores de cobertura se mantuvieron sin variación durante toda la primer etapa (figura 1a).

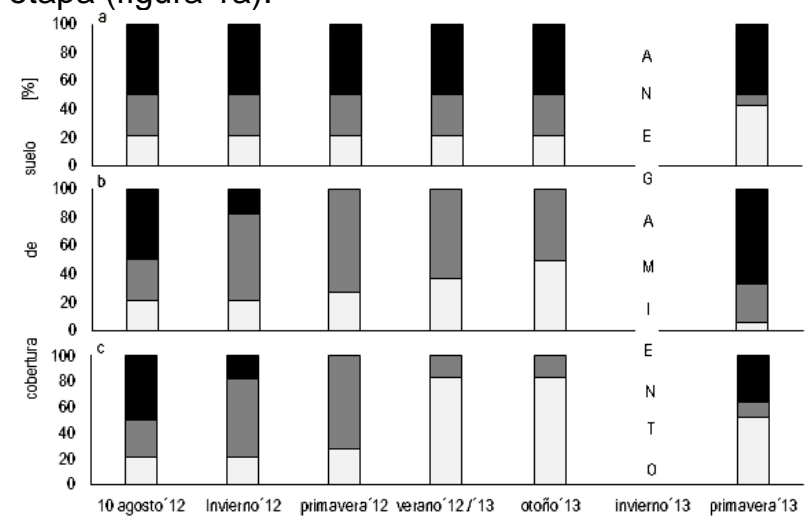

Fig. 1. Porcentaje de cobertura de suelo por estación climática en los tratamientos: (a) $P$ pastoreo continuo, (b) S: clausura sin cortes y (c) $\mathrm{C}$ : clausura con cortes bimestrales. $\square$ : suelo desnudo; $\square$ : suelo cubierto con especies no forrajeras y $\square$ : suelo cubierto con especies forrajeras.

Dentro de las clausuras antes de cumplirse los dos meses (muestreo de primavera 2012, figura $1 b$ y 1c), aún no se había realizado ningún corte en C. Todo el suelo estaba cubierto, en mayor parte con especies no forrajeras (73\%).

Durante el verano 2012-2013 en el tratamiento C las especies forrajeras cubrían mayor porcentaje de suelo que las no forrajeras.

En S, el grupo de especies no forrajeras tuvo mayor capacidad de crecimiento, llegando a cubrir el $63 \%$ de la superficie. A pesar de estas observaciones, no se detectaron diferencias estadísticas entre estos dos tratamientos (Kruskal Wallis, $p=0,2)$. En nuestro caso, con alturas de canopeo mayores a $50 \mathrm{~cm}$ y cierta competencia por luz, las especies colonizadoras resultaron ser más exitosas, mientras que la recuperación de especies de interés forrajero fue más lento.
Aunque no se registraron diferencias significativas entre los tratamientos (Kruskal Wallis, $p=0,2$ ) en otoño de 2013 , en el tratamiento $S$ las especies forrajeras abarcaron apenas la mitad de la superficie $(49 \%)$ respecto de C, donde se mantuvieron las proporciones de cobertura logradas en el verano.

Luego del anegamiento (primavera 2013), al final de la segunda etapa, la situación de los tres tratamientos fue muy disímil (Kruskal Wallis, $\mathrm{p}=0.0036$; figura 1). En el tratamiento $\mathrm{C}$, la cobertura de las especies no forrajeras era baja (pasó de 73 a casi $12 \%$ ), especialmente aquellas de porte erecto, cuyas yemas de ramificación y reproductivas se ubicaban por encima de la altura de corte $(7 \mathrm{~cm})$. La recuperación de la cobertura en el tratamiento $\mathrm{S}$ fue más lenta. Este tratamiento resultó el del mayor \% de suelo desnudo (67\%), consecuencia de la estructura del canopeo durante la clausura, donde, a pesar de que la vegetación no dejó suelo al descubierto, en la parte basal no existió un tapiz cerrado, sino que estaba formado por los tallos de las plantas que ganaron altura, en su mayoría especies no graminiformes. Aquí la recuperación de la vegetación fue menor que en $\mathrm{C}$, ya que las yemas de estos vegetales estuvieron más expuestas al pastoreo intenso. En esta situación final la cobertura del suelo con especies no forrajeras fue del $27 \%$ y con especies forrajeras, del 6\%. En el muestreo luego del anegamiento, el \% de SD del tratamiento $\mathrm{P}$ se mantuvo invariable y la cobertura con especies forrajeras (más del 42\%) fue mayor que con no forrajeras, que terminó con 7,5\% (figura 1a). Respecto a la situación inicial, la cobertura de forrajeras se duplicó, sin embargo, la única especie forrajera presente fue Cynodon dactylon.

Riqueza florística: Durante el período de estudio se determinaron 27 especies con amplia variedad en hábitos de crecimiento, ciclo, forma de reproducción y propagación. Un tercio de las especies son consideradas forrajeras (anexo) y una gran proporción son nativas (tabla 1).

Las especies exóticas mantuvieron una presencia constante en los tres tratamientos. Las no forrajeras solo aumentaron en el tratamiento C. Las especies forrajeras variaron en todos los tratamientos: aumentaron dentro de las clausuras (S y C), y disminuyeron en $\mathrm{P}$. De igual modo ocurrió con las especies nativas (tabla 1). La riqueza florística se redujo en $\mathrm{P}$, mientras que en las clausuras aumentó. En C se encontraron 16 especies en el primer muestreo y al final cuatro más: Alternathera philoxeroides, Oplismenopsis najada, Pluchea sagittalis, Hypochaeris microcephala, todas nativas y las dos primeras, excelentes forrajeras. Sin embargo, en $S$, el número de especies solo aumentó dos especies 
más. Al momento del último muestreo, en $\mathrm{P}$ había una sola especie forrajera, Cynodon dactylon, considerada regular en cuanto a su calidad.

Tabla 1: Número de especies presentes discriminadas por grupos de interés (G: graminiformes, L: latifoliadas, F: forrajeras, $\mathrm{N}$ : nativas, F: forrajeras), encontradas en la situación inicial (31 de agosto de 2012) y la situación final (13 de setiembre de 20213) y porcentajes relativos. P: pastoreo continuo, $\mathrm{S}$ : clausura sin cortes y C: clausura con cortes bimestrales.

\begin{tabular}{|c|c|c|c|c|c|c|}
\hline \multirow{2}{*}{$\begin{array}{l}\text { Grupos } \\
\text { de interés }\end{array}$} & \multicolumn{3}{|c|}{ Situación inicial } & \multicolumn{3}{|c|}{ Situación final } \\
\hline & $P$ & S & C & $P$ & S & C \\
\hline Especies & 17 & 15 & 16 & 13 & 17 & 20 \\
\hline № $G$ & 3 & 4 & 4 & 1 & 5 & 5 \\
\hline$\% G$ & 17,6 & 26,7 & 25 & 7,7 & 29,4 & 25 \\
\hline № L & 14 & 11 & 12 & 12 & 12 & 15 \\
\hline$\%$ L & 82,4 & 73,3 & 75 & 92,3 & 70,6 & 75 \\
\hline № F & 5 & 5 & 5 & 1 & 7 & 7 \\
\hline$\% \mathrm{~F}$ & 29,4 & 33,3 & 31,3 & 7,7 & 41,2 & 35 \\
\hline $\mathrm{N} N \mathrm{~N}$ & 14 & 11 & 12 & 10 & 13 & 16 \\
\hline$\% N$ & 88,2 & 73,3 & 75 & 76,9 & 76,5 & 80 \\
\hline № F & 3 & 4 & 4 & 3 & 4 & 4 \\
\hline$\% \mathrm{~F}$ & 17,7 & 26,7 & 25 & 23,1 & 23,5 & 20 \\
\hline
\end{tabular}

En invierno de 2012, 20 días después de iniciadas las clausuras, los dos primeros ejes del diagrama de dispersión del análisis de componentes principales absorbieron más del $74 \%$ de la varianza, separando a los censos $P$ de los $C$ y $S$ (figura 2a). Las especies Solanum glaucophyllum, Plantago sp., Hypochaeiris microcephala y Mimosa pellita estuvieron presentes en $\mathrm{C}$ y $\mathrm{S}$, pero no en $\mathrm{P}$, que tuvo mayor presencia de Rumex crispus. El 93,9\% de varianza explicada por los dos primeros ejes en el análisis correspondiente a primavera de 2012, refleja una gran similitud entre los sitios de muestreo y diferencia despreciable por tratamiento (figura $2 b$ ), lo que coincide con el hecho de que aún no se habían hecho cortes en las parcelas $C$.

En el verano 2012 - 2013, el PCA separó las parcelas $\mathrm{C}$ en los cuadrantes III y IV, pero cercanos al 2 do eje, con 47,8 y $24,2 \%$ de la variancia absorbidas por el 1 er y 2 do eje, respectivamente (figura 2c). A diferencia de las $\mathrm{S}$, las parcelas $\mathrm{C}$ no presentaban Xanthium cavallinesii ni Rumex crispus y estaban asociadas a una mayor presencia de Cynodon dactylon y Aster squamatus. Esto confirma diferencias causadas por los cortes, ya que aquellas especies con yemas por debajo de la altura de corte, aparecen en el tratamiento $\mathrm{C}$, mientras no se encuentran especies que no cuentan con yemas bajas.

(a)

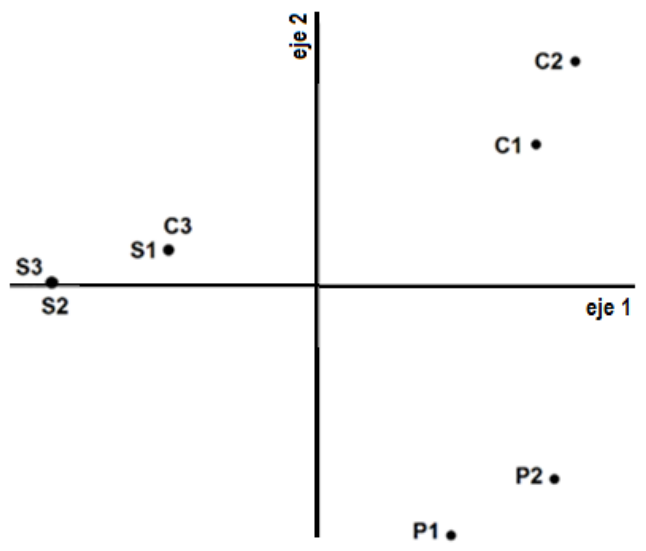

(b)



(c)

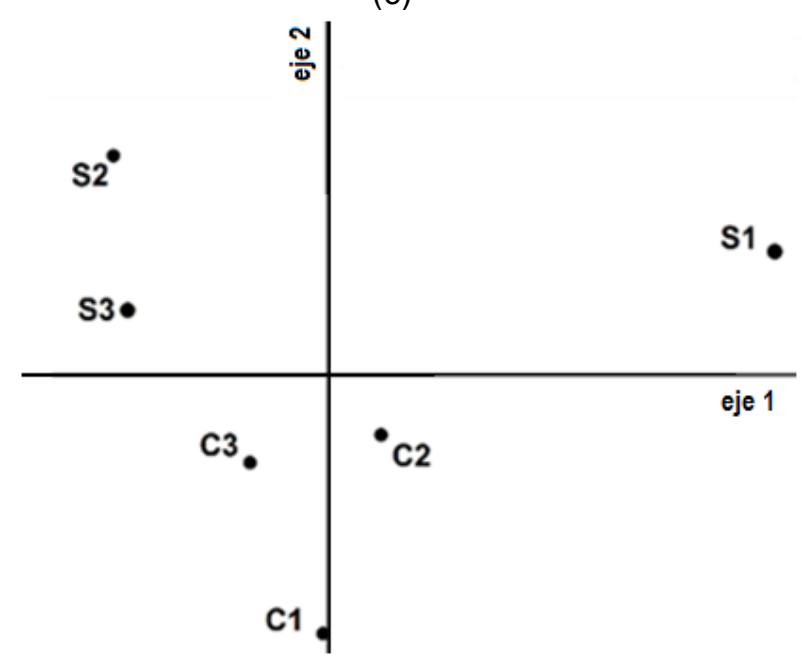


(d)
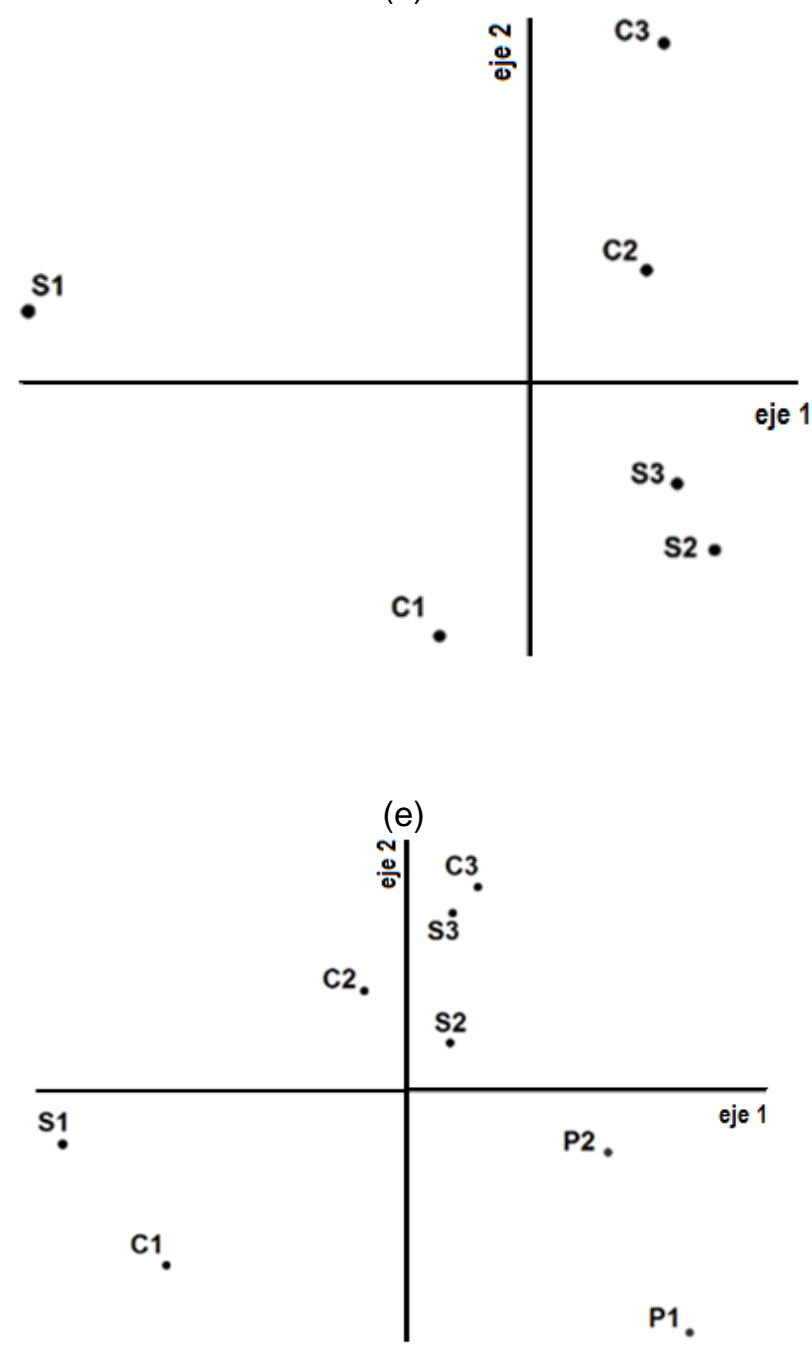

Fig. 2. Diagrama de dispersión del análisis de componentes principales (PCA) de los censos de vegetación para los tres tratamientos: $(P)$ con pastoreo continuo, (S) sin cortes y (C) con cortes bimestrales, por estación climática: (a) invierno 2012, (b) primavera 2012, (c) verano 2012-2013, (d) otoño 2013 y (e) primavera 2013.

A pesar de que la varianza del muestreo de otoño de 2013 explicada por los dos primeros ejes del PCA fue de $81,17 \%$ (figura $2 d$ ) y que para esta fecha se habían realizado tres cortes en las parcelas $\mathrm{C}$, el análisis PCA presentó un patrón de distribución que separa los tratamientos $S$ de $C$ para solo dos de tres sitios, distanciados sobre el eje 2. Se interpreta que las diferencias a causa de los cortes bimestrales sobre las especies primavero-estivales perdieron fuerza y que, por ello, vuelven a cobrar relevancia las diferencias por sitio. Distichlis spicata, Alternathera philoxeroides, Rumex crispus y Verbena gracilescens habían disminuido su área foliar acorde a la estación, que se corresponde al final de su ciclo, borrando los efectos que se habían expresado en la primavera y el verano anteriores. El último muestreo, en primavera de 2013, fue realizado durante el rebrote primaveral, luego del pastoreo intenso y anegamiento. Aunque no formaron un grupo compacto en el PCA (figura $2 e)$, las parcelas $P$ se agruparon a un extremo del eje 1 (4to cuadrante), mientras que las parcelas de los otros tratamientos ocuparon el resto del diagrama, manteniendo las cercanías por sitio. Los dos primeros ejes del análisis explicaron el $66,15 \%$ de la varianza total. Varias especies, como Paspalum dilatatum, Oplismenopsis najada, Distichlis spicata y Poa annua, Alternathera philoxeroides, Poligonum sp. y Taraxacum officinalis se encontraban en las clausuras y no estaban en P. Los efectos por descanso del pastoreo trascendieron el anegamiento. Sin embargo, dentro de las clausuras las diferencias en la riqueza florística logradas por ejecutar cortes bimestrales no se expresan en esta estación agroclimática en el diagrama PCA, sino que se vuelven a agrupar por sitio.

Altura de la vegetación y disponibilidad de materia seca: Al inicio de esta investigación, debido a que la vegetación del pastizal estaba por debajo de los $7 \mathrm{~cm}$ de altura, la MS fue nula en todos los tratamientos. En el tratamiento $\mathrm{P}$ se mantuvo así a causa de la fuerte presión de pastoreo sin descansos, donde la altura del tapiz siempre estuvo por debajo de los $7 \mathrm{~cm}$ de altura y, por consiguiente, no se cuentan con datos de MS para este tratamiento.

La altura en las parcelas de los otros tratamientos aumentó desde su clausura al pastoreo, en invierno de 2012 (figura 3). En el C la vegetación alcanzó su mayor altura en verano de 2012 y, a causa de los cortes, permaneció siempre por debajo de los $30 \mathrm{~cm}$, donde se observó un autosombreado despreciable en el canopeo. Por el contrario, en el tratamiento $S$ la altura de la vegetación fue aumentando hasta el verano, llegando a superar los $80 \mathrm{~cm}$ y luego decayó levemente, determinando que hubiera más autosombreado que en $\mathrm{C}$.



Fig. 3. Altura media de la vegetación (cm) en los 
tratamientos S (sin cortes, línea de guiones) y C (con cortes bimestrales, línea continua).

En las parcelas C, la mayor producción de MS se registró en primavera y a principios de verano (diciembre), mientras que en otoño e invierno la producción fue de menos de la mitad (figura 4a). En el tratamiento $\mathrm{S}$, la MS también aumentó hasta el verano 2012 - 2013 (figura 4b), tanto la viva (MS forrajera + MS no forrajera) como el material muerto en pie. A partir de entonces y hasta el otoño, la MS del material muerto continuó en aumento, mientras que la MS viva fue decayendo levemente en coincidencia con el fin de ciclo de la mayoría de las especies presentes, que en esta época pasaron al estado reproductivo, aumentaron su proporción tallo/hoja y comenzaron a senescer.

(a)

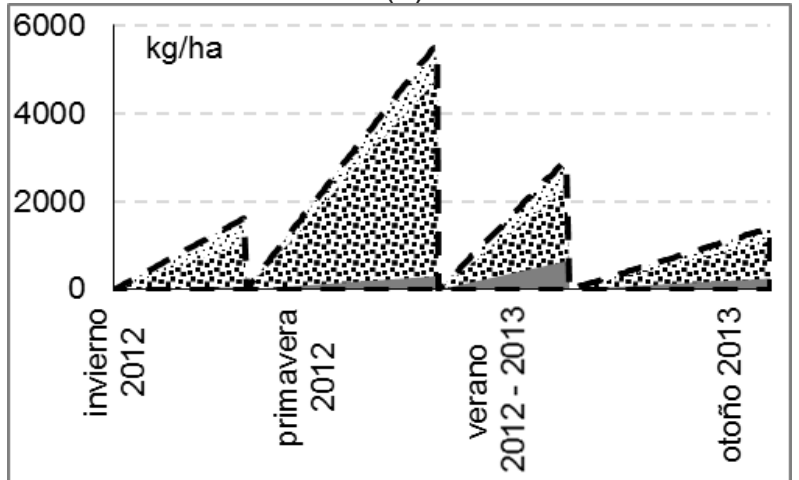

(b)

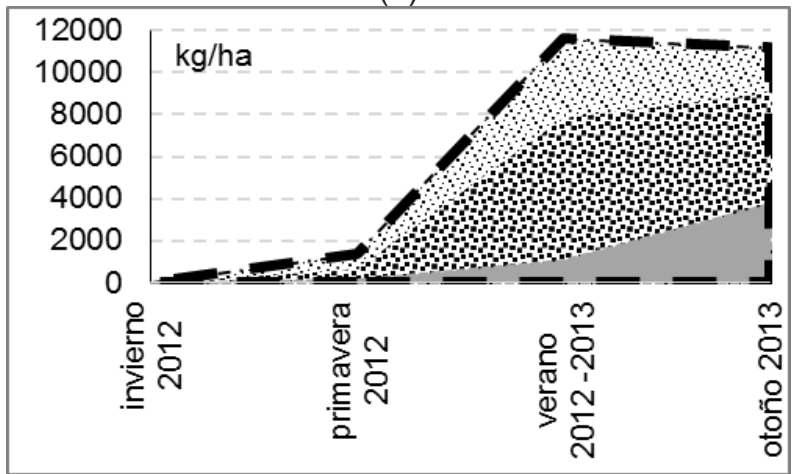

Fig. 4. MS acumulada por encima de los $7 \mathrm{~cm} \mathrm{(kg}$ ha $^{-1}$ ) en los tratamientos (a) C (con cortes bimestrales) y (b) $S$ (sin cortes) a lo largo del tiempo: MS total (línea de guiones), MS no forrajera圈, MS forrajera周, MS de biomasa muerta $\square$.

Una proporción relativamente importante de la MS viva total no correspondió a especies forrajeras, sino principalmente a Xanthium cavallinesii (abrojo grande), cuyo ciclo, comparado con el de las demás especies, presenta un crecimiento importante en verano, encontrándose dentro de la proporción de MS viva hasta otoño, cuando empieza a senescer y finalmente pasa a estar en la proporción de MS muerta. Entre las especies forrajeras presentes, predominó Cynodon dactylon.

La diferencia entre los valores de MS viva total en verano entre $S\left(10533 \mathrm{~kg} \mathrm{ha}^{-1}\right)$ y C $\left(2237 \mathrm{~kg} \mathrm{ha}^{-1}\right)$ fue de $8296 \mathrm{~kg}$, a favor de S. Esta diferencia de cosecha se obtuvo luego de haber realizado solo dos cortes bimestrales en C (a mediados de octubre y de diciembre). En relación a la MS viva forrajera, la diferencia es de aproximadamente la mitad (4667 kg MS ha-1) a favor de S, cuyo valor corresponde a una sola cosecha efectuada en verano $\left(6733 \mathrm{~kg} \mathrm{ha}^{-1}\right)$. Sin embargo, en C el corte realizado en ese momento es el tercero. Por lo tanto, la suma de las cosechas de MS forrajera acumulada en $C$ hasta este momento es de 7417 $\mathrm{kg} \mathrm{ha}^{-1}$. En el tratamiento $C$ se cosecharon $684 \mathrm{~kg}$ $\mathrm{ha}^{-1}$ de MS forrajera adicionales. De este modo el área con cortes bimestrales produjo un $22,5 \%$ más de MS forrajera que su par clausurado sin cortes (S).

\section{DISCUSIÓN}

Este pastizal albergó herbívoros, por lo tanto el pastoreo se considera parte del sistema y un elemento necesario para mantener las características y a sus especies nativas [24].

La recuperación inicial de la cobertura a partir del grupo de especies no forrajeras, revela la degradación del pastizal a causa de la alta presión de pastoreo previa, considerando que el manejo y la historia de eventos y disturbios de un pastizal repercuten en la disponibilidad de forraje y en su integridad [5] [28].

Las especies pueden agruparse de acuerdo a sus estrategias en el aprovechamiento de la luz y su respuesta al pastoreo [29] y, en nuestra experiencia, el manejo con cortes bimestrales favoreció a las gramíneas, que representan a casi la totalidad de las especies forrajeras. Aquellas especies con aptitudes invasoras, colonizadoras o características que les permiten adaptarse al sobrepastoreo (Phyla canescens, Hypochaeris microsephala, Solanum glaucophyluam, Mimosa pellita, Xanthium cavalineessi), y habían iniciado la cobertura de los sectores de suelo desnudo, redujeron marcadamente su presencia, ya que sus yemas se encuentran por encima de la altura de corte. Simultáneamente, las alturas relativamente bajas del canopeo (entre 7 y $30 \mathrm{~cm}$ ) favorecieron la aparición y el crecimiento de especies de bajo porte o estoloníferas, que en su mayoría fueron de buena a excelente calidad forrajera [10]. Por lo tanto, en el tratamiento $\mathrm{C}$, a pesar que al principio el suelo se había cubierto principalmente con especies no forrajeras, la recuperación se llevó a cabo mayoritariamente a partir de especies forrajeras $(52 \%)$, principalmente estoloníferas o con yemas de renuevo bajas, tanto de la familia de las Poaceae (Paspalum dilatatum, Poa annua, 
Paspalum sp., Oplismenopsis najada), como también Polygonum punctatum y Alternathera phyloxeroides. Esta última especie, presente en la recuperación del tratamiento $\mathrm{C}$, es excelente forrajera, ya que contiene un $20 \%$ de proteína bruta y $70 \%$ de digestibilidad, valores comparables a los de la alfalfa, Medicago sativa [10]. El manejo con cortes bimestrales permitiría el control de las hierbas menos deseables y favorecería el objetivo productivo de lograr una riqueza botánica en que las especies de mayor aceptabilidad sean las que estén presentes en mayor proporción [30].

Además del efecto por cortes, es probable que haya existido también un efecto por el anegamiento. Según estudios de Otondo et al. [29], en el pastizal de la cuenca hídrica del Salado las inundaciones favorecen a las especies graminoides, perjudicando a las dicotiledóneas, lo cual revierte temporalmente el deterioro de la vegetación provocado por el exceso de pastoreo y mejorando la cantidad y calidad del forraje disponible. Esto se verifica siempre y cuando la acumulación de agua permita la llegada de luz al suelo. Afirman que los pastizales que coevolucionaron con eventos de anegamiento frecuentes, presentan una gran cantidad de especies que se benefician con la misma. Estos autores concuerdan con que los efectos dependerán también en gran medida del manejo del pastoreo (carga y frecuencia) y de las condiciones climáticas imperantes luego de que se retire el agua. Moreno-Casasola et al. [31] afirman que los principales factores físicos involucrados en mantener la diversidad vegetal de los humedales son la micro-topografía, el nivel y tiempo de inundación, la salinidad en suelo y agua.

El sobrepastoreo en el humedal alto-andino de Perú supera la capacidad de regeneración de la vegetación [32], que durante la estación seca no alcanza a cubrir el suelo y se acentúa la degradación hídrica y eólica del mismo. Con cargas continuas muy altas y falta de técnicas adecuadas que eviten la eliminación de la cobertura vegetal, además de desaparecer gran parte de la vegetación propia, el suelo pierde características como capacidad de filtración y retención de agua. El conocimiento de las relaciones entre variables estructurales, como por ejemplo biomasa aérea y altura, que determinan el tamaño de bocado y accesibilidad por parte del animal, facilita la implementación práctica de las decisiones de manejo [33].

En los ecosistemas adaptados al pastoreo es compatible la explotación y conservación de los recursos y ciertos niveles de pastoreo son fundamentales para conservar la diversidad [34]. Sin embargo, se pueden alcanzar estados de degradación tanto por exceso como por abandono del pastoreo. Si la intensidad de explotación se acentúa, el ecosistema entra en crisis y puede llevar a la pérdida de especies, donde llegada a cierta situación, el ecosistema ya no tiene capacidad de retorno a las situaciones anteriores, al menos, a la escala temporal de una explotación ganadera [27]. Jacobo y Rodríguez [35] afirman que también la falta de disturbios degrada la vegetación.

A pesar que bajo nuestras condiciones experimentales, dentro de las clausuras no se detectaron diferencias estadísticas en cuanto a presencia de especies, los resultados concuerdan con Travieso-Bello et al. [28], quienes hallaron que la mayor riqueza de especies se verifica bajo situaciones intermedias de disturbio y que los valores más altos de riqueza florística se obtienen con valores intermedios respecto a la biomasa. Seguramente se hubieran encontrado especies con yemas a diferentes alturas si, en lugar de someter al pastizal a cortes bajos, se lo hubiera pastoreado de forma moderada, ya que Paruelo et al. [36] suponen que este manejo promovería un aumento de la diversidad vegetal. Esto coincide con las afirmaciones de Deregibus [37] y Jacobo y Rodríguez [35], quienes sostienen que los pastizales de regiones húmedas de nuestro país han evolucionado con bajas presiones de pastoreo y mantienen la máxima diversidad de especies bajo esas condiciones. Collins [38] afirma que ante un patrón regional de disturbios repetitivos (el fuego en su caso), existe un grado sorprendente de cambio direccional en la vegetación. Por lo tanto, sería esperable que los cambios a largo plazo en la vegetación respondan al patrón de pastoreo como disturbio modelador, y que un manejo semejante al pastoreo de herbívoros nativos mantenga una dirección semejante respecto a los cambios de la comunidad florística.

La producción de MS del pastizal de albardón estudiado coinciden con resultados similares en otras experiencias de este tipo [36], aunque puede ser considerada alta si se la compara con otros humedales, como los altoandinos de la provincia de Candarave, departamento de Tacna, Perú, donde Condori [39] reportó $526,39 \mathrm{~kg} \mathrm{ha}^{-1}$ y Alvarado Calderón [40], $577,34 \mathrm{~kg} \mathrm{ha}^{-1}$, para un corte en la época lluviosa; mientras que Luna et al., [41] registraron hasta $6860,7 \mathrm{~kg} \mathrm{ha}^{-1}$, en Bolivia.

\section{CONCLUSIONES}

El pastoreo continuo, prolongado y con altas cargas afecta la riqueza florística de este pastizal, eliminando aquellas especies con buena calidad forrajera y favoreciendo la expansión de otras no palatables. Se comprobó que bajo estas condiciones, la cosmopolita Cyndon dactylon 
desplaza a gramíneas nativas propias del humedal y gran parte del suelo permanece desnudo.

La clausura al pastoreo, bajo nuestras condiciones experimentales, aumentó la riqueza florística con especies nativas y forrajeras de crecimiento primavero-estival, en mayor proporción donde se aplicaron cortes bimestrales. Los descansos, manteniendo al canopeo por debajo de los $30 \mathrm{~cm}$, permitieron una recuperación con mayor riqueza florística y más rápida del pastizal luego del intenso pastoreo y el anegamiento. El tratamiento sin pastoreo ni cortes favorece el aumento de la riqueza florística inicial. Sin embargo, también aumenta significativamente la fracción de material muerto en pie, situación atribuible al autosombreado dentro del canopeo.

La producción de MS también fue mayor con el manejo de cortes bimestrales, cuyos valores sugieren que dos meses es un tiempo adecuado para que el pastizal logre desarrollar una biomasa máxima o cercana a la máxima. Este manejo también favoreció otros bienes y servicios, como la flora apícola nativa, especies vegetales que constituyen la dieta de fauna nativa, etc.

$\mathrm{Ni}$ los descansos prolongados ni la ausencia de disturbios favorecerían la resiliencia del pastizal, manteniendo su riqueza, si no que los disturbios dentro de cierto rango juegan un importante papel en mantener elevada la diversidad y su resiliencia. Se destaca que el efecto acumulado del pastoreo selectivo con alta presión de los últimos años, no impide la rápida recuperación del pastizal bajo condiciones adecuadas. Los valores de MS total del pastizal en el período primavera - verano otoño, con descansos bimestrales, sugieren que es un tiempo adecuado para que el pastizal logre acumular una biomasa óptima. Sin embargo, es de esperar que con ensayos más prolongados al nuestro se registre una dinámica distinta en cuanto a las variables analizadas con cambios más importantes y de diferencias más significativas.

El efecto del pastoreo selectivo con alta presión de los últimos años no impide la rápida recuperación del pastizal cuando se aplica el pastoreo adecuado tanto en la presión como la frecuencia.

\section{AGRADECIMIENTOS}

Agradezco al INTA, ya que financió los estudios y al productor por permitir hacer el experimento en su campo.

\section{REFERENCIAS BIBLIOGRÁFICAS}

[1] Vizia, C., Spiaggi, E., Stancich, E., Donadille, G., Postma, J., Prol, L., Romano, M., Kandus, P. y Minotti, P. Humedales del Paraná. Biodiversidad, usos y amenazas en el Delta Medio: Inercia Comunicaciones, 67 p., Rosario, 2010

[2] Sepulcri, M.G.; Pizarro, M.J.; Flamenco, E.; Herrera, M.; Borus, J.; Giordano, L. Cartografía de susceptibilidad hídrica en el delta del río Paraná: Revista RIA - INTA, 2012. Disponible en: http://ria.inta.gov.ar/?p=2002 [consultado el 17 de octubre de 2014]

[3] Kalesnik, F. y Quintana, R.. El Delta del Río Paraná como un mosaico de humedales. Caso de estudio: La Reserva de Biosfera MAB-UNESCO 'Delta del Paraná': Revista UnG - Geociências V.5, N.1.pag.: 22-37, 2006. Disponible en: http://revistas.ung.br/index.php/geociencias/artic le/viewFile/92/185 [consultado el 21 de octubre de 2014]

[4] Torres Robles, S. S. Variación geográfica de la composición y riqueza de plantas vasculares en los talares bonaerenses y su relación con el clima, sustrato, estructura del paisaje y uso [PhD Thesis]. Facultad de Ciencias Naturales y Museo, Universidad Nacional de La Plata, Argentina, 2009. Disponible en: http://hdl.handle.net/10915/55171 [consultado el 24 de noviembre de 2016]

[5] Quintana, R. D. Lineamientos para una ganadería ambientalmente sustentable en el Delta del Paraná: 1a ed. - Buenos Aires: Fundación para la Conservación y el Uso Sustentable de los Humedales. $24 \times 17 \mathrm{~cm}$. 1. Ganadería. 2. Humedales. I. 128 pp., 2014.

[6] Silva Busso, A. Aportes al conocimiento de la Geología del Subsuelo del Delta del Río Parana': Proyecto: Simulación y Predicción del Crecimiento del Frente del Delta del Río Paraná - PICT 802/OC-AR - Instituto Nacional del Agua, Subsecretaría de Recursos Hídricos, Secretaría de Obras y Servicios Públicos, Proyecto LHA 235Informe Laboratorio de Hidráulica 03-235-04, $2004 . \quad$ Disponible en: http://laboratorios.fi.uba.ar//mm/informes/it delta $\mathrm{g}$ eologia sep04.pdf [consultado el 22 de noviembre de 2014][7] Aceñolaga, P. G.; Povedano, H.E.; Adriana S. Manzano, A. S.; Muñoz, J. de D.; Areta, J. I.; Ronchi Virgolini, A. L. Biodiversidad del Parque Nacional Pre-Delta - Temas de la Biodiversidad del Litoral fluvial argentino: INSUGEO, Miscelánea, 12. Pag.: 169 - 184, 2004. [8] Bó, R. F. Situación ambiental en la Ecorregión Delta e Islas del Paraná en Ecorregión Delta e Islas del Paraná en La Situación Ambiental Argentina: Grupo de Investigaciones en Ecología de Humedales (GIEH). Laboratorio de Ecología Regional. Departamento de Ecología, Genética y Evolución. Facultad de Ciencias Exactas y Naturales (FCEN), Universidad de Buenos Aires, Argentina, 2005. Disponible en: http://www.fvsa.org.ar/situacionambiental/delta.pdf [consultado el 26 de marzo de 2016] 
[9] Kandus, P.; Morandeira, N. y Schivo, F. (eds) Bienes y Servicios Ecosistémicos de los Humedales del Delta del Paraná: Fundación Humedales / Wetlands International. Buenos Aires, Argentina, 2010.

[10] Rossi, C. A.; De Magistris, A.A.; González, G. L.; Carou, N. E.; De Loof, E. P. Plantas de interés ganadero de la región del bajo Delta del Paraná, Argentina: 1ra ed. Lomas de Zamora, Editorial UNLZ, Facultad de Ciencias Agrarias. E-book, $2014 . \quad$ Disponible en: https://www.researchgate.net/publication/2691644 38 PLANTAS DE INTERES GANADERO DE L A REGION DEL BAJO DELTA DEL PARANA ARGENTINA Plantas forrajeras herbaceas y le nosas Valor nutritivo del forraje natural Maleza s y plantas toxicas para el ganado Biodive [consultado el 22 de julio de 2016]

[11] Bavera, G. A. Equivalencias Ganaderas: Recopilación para el Curso de Producción Bovina de Carne, FAV UNRC, 2006. Disponible en: http://www.produccion-

animal.com.ar/produccion y manejo

pasturas/pastoreo\%20 sistemas/70equivalencias ganaderas.pdf [consultado el 12 de noviembre de 2017]

[12] Quintana, R. D. y Bó, R. F. Humedales del Delta del Paraná: Inventario de los humedales de Argentina: sistemas de paisajes de humedales del corredor fluvial Paraná Paraguay. Secretaría de Ambiente y Desarrollo Sustentable de la Nación. Proyecto GEF 4206 PNUD ARG 10/003. Edición literaria a cargo de Laura Benzaquén et.al. - 1a ed. $30 \times 21 \mathrm{~cm} .376$ pp., 2013.

[13] Connell, J.H. Diversity in tropical rain forests and coral reefs. Science 199:13021310, 1978.

[14] Magnano, A.; Vicari, R.; Astrada, E.; Quintana, R. Ganadería en humedales. Respuestas de la vegetación a la exclusión del pastoreo en tres tipos de ambientes en un paisaje del Delta del Paraná: RASADEP. 5. 137-148, 2013.

[15] Egler, F. E.; Vegetatio Acta Geobot. Volume 4, Issue 6, pp 412-417. Online ISSN: 1573 - 5052. 1954.

[16] Martínez Ramos, M. Grupos funcionales: Capital natural de México, vol. I: Conocimiento actual de la biodiversidad. Conabio, México. Pag.: 365-412, 2008

[17] Minotti, P. G.; Baigún, C.; Kandus, P.; Quintana, R. D.; Borro, M.; Schivo, F.; Morandeira, N.; Gramuglia, P. y Brancolini, F. Servicios Ecosistémicos en la Ecorregión del Delta del Paraná: Consideraciones sobre Usos y Tenencias, y Criterios para su Conservación: Estrategias Integradas de Mitigación y Adaptación a Cambios Globales. Edición: Fernández Reyes, L.; Volpedo, A. V.; y Pérez Carrera, A. Buenos Aires, Argentina. ISBN: 978-987-96413-9-2, 2009.
[18] Madanes, N. Efecto del fuego en un juncal de Schoenoplectus californicus en la Región del Delta del Río Paraná: Delta del Paraná: Historia, presente y futuro. Volumen 2 - Trabajos completos Simposio Científico Académico Delta del Paraná San Fernando, Argentina, 4 y 5 de octubre, 2010. Editores: Kalesnik, F.; Vicari, R. e Iribarren, L. (C) UNESCO 2013, 2010.

[19] Carambula, M. Pasturas naturales mejoradas: VETERINARIA. Sociedad de Medicina Veterinaria del Uruguay, Año LXVI Vol. 41 No 161-162. Enero - Junio de 2006. Uruguay. 524 pp., 1996. Disponible en: http://www.revistasmvu.com.uy/revistas/numero16 1-162.pdf [consultado el 23 de enero 2016]

[20] Roman, L.; Cocco, M.; Messina N.; Barreto C; Romero G. Fuser, C. Productividad del pastizal natural en la Estancia El Espinillo durante 2016: Centro Regional Entre Ríos Estación Experimental Agropecuaria Concordia. Sitio Argentino de Producción Animal, 2016. Disponible en: $\quad$ http://www.produccionanimal.com.ar/produccion y manejo pasturas/pa sturas\%20naturales/234-

productividad del pastizal natural.pdf [consultado el 02 de octubre de 2017]

[21] Di Rienzo, J.A.; Gonzalez, L.; Casanoves, F.; Tablada, M. Balzarini, M. InfoStat - Software estadístico: Registro Dirección Nac. Derecho de Autor, obra de software, $\mathrm{N} \cong$ 960318, 2011. Disponible en: http://www.infostat.com.ar [consultado el 16 de noviembre de 2016]

[22] Braun-Blanquet, J. Fitosociología. Blume Ediciones, Madrid. España. 820 pp., 1979.

[23] van der Maarel, E. Transformation of coverabundance values in phytosociology and its effects on community similarity: Vegetatio 39: 97-114, 1979.

[24] Mc Cune, B. y Mefford, m. J. PC-ORD. Multivariate Analysis of Ecological Data: Version 6. MjM Software, Gleneden Beach, Oregon, U.S.A, 2011.

[25] Zuloaga, F.; Morrone, O. y Belgrano, M. (eds.) Catálogo de las Plantas Vasculares del Cono Sur (Argentina, sur de Brasil, Chile, Paraguay y Uruguay): Monogr. Syst. Bot. Missouri Bot. Gard. 107, 2008.

[26] Mendoza P. y Lascano, C. Mediciones en la pastura en ensayos de pastoreo: Evaluación de pasturas con animales. Alternativas metodológicas. Lascano C, Pizarro E. editors. Red Internacional de Evaluación de Pastos Tropicales (RIEPT); CIAT.: pag.:143-165. Cali, Colombia, 1986.

[27] Rebollo, S. y Gómez-Sal, A. Aprovechamiento sostenible de los pastizales: Ecosistemas_Revista Científica y Técnica de Ecología y Medio Ambiente_Año XII, No $3 / 2003$ septiembrediciembre, 2003. Disponible en: 
http://www. aeet.org/ecosistemas/033/investigacion 7.htm [consultado el 13 de diciembre de 2017] [28] Travieso-Bello, A. C.; Moreno-Casasola, P.; Campos, A. Efecto de diferentes manejos pecuarios sobre el suelo y la vegetación en humedales transformados a pastizales: Interciencia, vol. 30, núm. 1, enero, 2005.: 12-18. Asociación Interciencia, Caracas, Venezuela, $2005 . \quad$ Disponible en: http://www.redalyc.org/articulo.oa?id=33910003 [consultado el 22 de agosto de 2012]

[29] Otondo, J., Casal, A., Coria, J. y Martinefsky, M. Respuesta de los suelos y pastizales después de la inundación: Sitio Argentino de Producción Animal. INTA Cuenca Informa 02.12.14. Chacra Experimental Integrada Chascomús (MAA-INTA), Argentina, $2014 . \quad$ Disponible en: http://www.produccionanimal.com.ar/inundacion/99-Respuesta.pdf [consultado el 13 de diciembre de 2017]

[30] Senra, A. Índices para controlar la eficiencia y sostenibilidad del ecosistema del pastizal en la explotación bovina: Revista Cubana de Ciencia Agrícola_39. ISSN: 0034-7485, 2005. Disponible en:

http://www.redalyc.org/articulo.oa?id $=1930178520$ 02 [consultado el 13 de diciembre de 2017]

[31] Moreno-Casasola, Patricia et al..Composición florística, diversidad y ecología de humedales herbáceos emergentes en la planicie costera central de Veracruz, México_Bol. Soc. Bot. Méx 2010, n.87 - pp.29-50. ISSN 0366-2128, 2010. Disponible en: http://www.scielo.org.mx/scielo.php?script=sci artt ext\&pid=S0366$21282010000200003 \&$ Ing =es\&nrm=iso [consultado el 20 de febrero de 2018]

[32] Gil Mora, Juan Eduardo Bofedal: Humedal altoandino de importancia para el desarrollo de Cusco. 2011

[33] Saldanha, S.; Boggiano, P.; Cadenazzi, M. Oferta de forraje, producción y composición de una pastura de Lolium perenne: Agrociencia Uruguay - Volumen 16 1:150-159 - enero/junio 2012, 2012.

[34] Marty, J. T. Effects of Cattle Grazing on Diversity in Ephemeral Wetlands: Conservation Biology 19. Pag.: 1626-1632, 2005. Disponible en: http://onlinelibrary.wiley.com/doi/10.1111/j.15231739.2005.00198.x/abstract [consultado el 20 de febrero de 2016]
[35] Jacobo, E. y Rodríguez, A. Valorización de Pastizales Naturales en Ambientes Húmedos. Indicadores de Sustentabilidad: $5^{\circ}$ Congreso de la Asociación Argentina para el Manejo de los Pastizales Naturales, Corrientes. Sitio Argentino de Producción Animal, 2009. Disponible en: http://www.produccion-

animal.com.ar/produccion y manejo pasturas/pa sturas\%20naturales/163-Valorizacion.pdf

[consultado el 10 noviembre de 2014]

[36] Paruelo, J.M.; Piñeiro, G.; Altesor, A. I.; Rodríguez, C. y Oesterheld, M. Cambios Estructurales y Funcionales Asociados al Pastoreo en los Pastizales del Río de la Plata: INIA La Estanzuela (LE), 2004.

[37] Deregibus, V.A. Importancia de los pastizales naturales en la República Argentina: situación presente y futura: Revista Argentina de Producción Animal 8. Pag.: 67-78, 1988.

[38] Collins, S.L.; Disturbance frequency and community stability in native tallgrass prairie. The American Nat. 155(3):311-325., 2000.

[39] Condori, F. Evaluación de la Producción Vegetal Forrajera de los Humedales Altoandinos de la Provincia de Candarave. [Graduate Thesis]. UNJBG/FCAG/EMVZ, Tacna - Perú. 76pp.

Facultad de Ciencias Naturales y Museo, Universidad Nacional de La Plata, Argentina, $2008 . \quad$ Disponible en: http://naturalis.fenym.unlp.edu.ar/repositorio/ docu mentos/tesis/tesis 1041.pdf [consultado el 18 octubre de 2014]

[40] Alvarado Calderón, C. O. Evaluación de Pastizales Naturales de los Humedales Altoandinos en Época de Lluvia de la Provincia de Candarave, Departamento de Tacna 2012 [Graduate Thesis] Universidad Nacional Jorge Basadre Grohman de Tacna, Facultad de Ciencias Agropecuarias, Escuela Académico Profesional de Medicina Veterinaria y Zootecnia. Tacna. Av. Miraflores s/n, 2012.

[41] Luna D, Alzérreca $H$, Prieto G, Céspedes J. Estudio de capacidad de carga de los bofedales para la cría de alpacas en el sistema T.D.P.S. Bolivia: Primera edición. Editorial Plural editores. Pg. 55, 56, 57, 58. La Paz, Bolivia, 2001. Disponible en: http://www2.congreso.gob.pe/sicr/cendocbib/con4 uibd.nsf/DD6CD3E44455A67A05257C6E005067 A5/\$FILE/Tesis Evaluaci\%C3\%B3n de Pastizale s Naturales Humedales.pdf [consultado el 18 de septiembre de 2018] 
ANEXO: Especies encontradas en la comunidad bajo estudio y sus características relevantes: familia botánica; vía fotosintética; tipo de reproducción (S: sexual, A: asexual); ciclo de vida (a: anual, b: bienal, p; perenne); calidad forrajera (t: tóxica, n: no forrajera, r: regular, b: buena, e: excelente); origen nativo; aptitud apícola y medicinal.

\begin{tabular}{|c|c|c|c|c|c|c|c|c|c|}
\hline Características & $\begin{array}{l}\stackrel{\widetilde{\sigma}}{\overline{\bar{E}}} \\
\stackrel{\widetilde{\sigma}}{\mathbb{\sigma}}\end{array}$ &  & 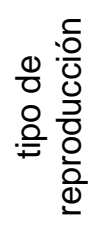 & $\frac{0}{ㅇ ㅡ ㄴ ~}$ & 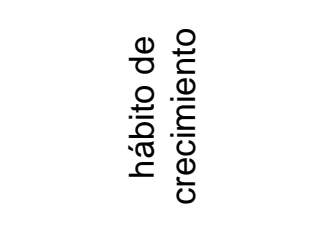 &  &  & $\begin{array}{l}\frac{\pi}{0} \\
\frac{0}{0} \\
\frac{0}{\sigma}\end{array}$ & 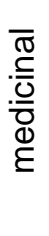 \\
\hline Xanthium cavallinesii & Asteraceae & C3 & $S$ & $\mathrm{a}$ & erguido & $\mathrm{t}$ & $\mathrm{x}$ & $x$ & $x$ \\
\hline Polygonum sp. & Polygonaceae & C3 & $\mathrm{S}, \mathrm{A}$ & $\mathrm{p}$ & erguido y apoyante & $r$ & $x$ & $x$ & $x$ \\
\hline Cynodon dactylon & Poaceae & C4 & $S, A$ & $p$ & rastrero & $r$ & & & \\
\hline Paspalum sp. & Poaceae & C4 & $\mathrm{S}, \mathrm{A}$ & $\mathrm{p}$ & erguido & b & $x$ & $x$ & \\
\hline $\begin{array}{l}\text { Phyla canescens } \\
\text { Oplismenopsis }\end{array}$ & Verbenaceae & C3 & $\mathrm{S}, \mathrm{A}$ & $\mathrm{p}$ & rastrero & $\mathrm{n}$ & $x$ & $x$ & $\mathrm{x}$ \\
\hline $\begin{array}{l}\text { najada } \\
\text { Eryngium }\end{array}$ & Poaceae & C4 & $\mathrm{S}, \mathrm{A}$ & $\mathrm{p}$ & rastrero o apoyante & e & $x$ & $x$ & \\
\hline chubutense & Apiaceae & C3 & S & $\mathrm{p}$ & erguido & $\mathrm{n}$ & $x$ & $x$ & \\
\hline Pluchea sagittalis & Asteraceae & C3 & $S$ & $\mathrm{p}$ & erguido & $\mathrm{n}$ & $x$ & $x$ & $x$ \\
\hline Mimosa sp. & Fabaceae & C3 & $S$ & $\mathrm{p}$ & erguido & $\mathrm{n}$ & $x$ & $\mathrm{x}$ & \\
\hline Dipsacus sativus & Asteraceae & C3 & $S$ & $b, p$ & erguido & $n$ & & $\mathrm{x}$ & \\
\hline $\begin{array}{l}\text { Acmella decumbens } \\
\text { Verbena }\end{array}$ & Asteraceae & C3 & $\mathrm{S}, \mathrm{A}$ & $\mathrm{p}$ & rastrero & $\mathrm{n}$ & $x$ & & $\mathrm{x}$ \\
\hline gracilenscens & Verbenaceae & C3 & $S$ & $\mathrm{p}$ & erguido & $n$ & $\mathrm{X}$ & $\mathrm{x}$ & \\
\hline Erigeron tweediei & Asteraceae & C3 & $S, A$ & $p$ & erguido & $\mathrm{n}$ & $x$ & & \\
\hline Jaborosa integrifolia & Solanaceae & C3 & $\mathrm{S}, \mathrm{A}$ & $\mathrm{p}$ & erguido & $\mathrm{n}$ & $x$ & & \\
\hline $\begin{array}{l}\text { Trifolium repens } \\
\text { Hypochaeris }\end{array}$ & Fabaceae & C3 & $S, A$ & $\mathrm{p}$ & rastrero & $b$ & & $x$ & $x$ \\
\hline $\begin{array}{l}\text { microcephala } \\
\text { Solanum }\end{array}$ & Asteraceae & C3 & $S$ & $\mathrm{p}$ & erguido & $\mathrm{n}$ & $x$ & $x$ & $x$ \\
\hline glaucophyllum & Solanaceae & C3 & $S, A$ & $\mathrm{p}$ & erguido & $\mathrm{t}$ & $x$ & & \\
\hline Distichlis spicata & Poaceae & C4 & $S, A$ & $\mathrm{p}$ & rastrero & $r$ & $x$ & & \\
\hline Poa annua & $\begin{array}{l}\text { Poaceae } \\
\text { Plantaginacea }\end{array}$ & C3 & $\mathrm{S}, \mathrm{A}$ & $\mathrm{a}$ & erguido & e & & & \\
\hline Plantago myosuros & e & C3 & $S$ & $\mathrm{p}, \mathrm{a}$ & arrosetado & $r$ & $x$ & $x$ & $x$ \\
\hline $\begin{array}{l}\text { Taraxacum officinale } \\
\text { Symphyotrichum }\end{array}$ & Asteraceae & C3 & S & $\mathrm{p}$ & arrosetado & $\mathrm{n}$ & & $x$ & $x$ \\
\hline squamatum & Asteraceae & C3 & S & $p$ & erguido & $\mathrm{n}$ & $x$ & $x$ & $x$ \\
\hline Malvella leprosa & Malvaceae & C3 & $S$ & $\mathrm{p}$ & rastrero & $\mathrm{n}$ & $x$ & & \\
\hline $\begin{array}{l}\text { Portulaca sp. } \\
\text { Alternanthera }\end{array}$ & $\begin{array}{l}\text { Portulacaceae } \\
\text { Amaranthacea }\end{array}$ & C3 & S, A & & postrado & $\mathrm{n}$ & $x$ & $x$ & \\
\hline philoxeroides & & C3 & S, A & $\mathrm{p}$ & apoyante y rastrero & e & $\hat{\lambda}$ & A & \\
\hline $\begin{array}{l}\text { Rumex crispus } \\
\text { Lepidium didymum }\end{array}$ & $\begin{array}{l}\text { Polygonaceae } \\
\text { Brassicaceae }\end{array}$ & $\begin{array}{l}\text { C3 } \\
\text { C3 }\end{array}$ & $\begin{array}{c}S, A \\
S\end{array}$ & $\begin{array}{l}\mathrm{p} \\
\mathrm{a}\end{array}$ & $\begin{array}{l}\text { erguido } \\
\text { arrosetado }\end{array}$ & $\begin{array}{l}\mathrm{n} \\
\mathrm{n}\end{array}$ & $\mathrm{x}$ & $x$ & \\
\hline
\end{tabular}


